\title{
A CLASS OF LINEAR VISCOELASTIC MODELS BASED ON BESSEL FUNCTIONS
}

\author{
IVANO COLOMBARO ${ }^{1}$, ANDREA GIUSTI $^{2}$, AND FRANCESCO MAINARDI $^{3}$
}

\begin{abstract}
In this paper we investigate a general class of linear viscoelastic models whose creep and relaxation memory functions are expressed in Laplace domain by suitable ratios of modified Bessel functions of contiguous order. In time domain these functions are shown to be expressed by Dirichlet series (that is infinite Prony series). It follows that the corresponding creep compliance and relaxation modulus turn out to be characterized by infinite discrete spectra of retardation and relaxation time respectively. As a matter of fact, we get a class of viscoelastic models depending on a real parameter $\nu>-1$. Such models exhibit rheological properties akin to those of a fractional Maxwell model (of order 1/2) for short times and of a standard Maxwell model for long times.
\end{abstract}

\section{INTRODUCTION}

The purpose of this paper is to introduce a rheological characterization of a peculiar class of models generalizing with infinite discrete spectra of retardation and relaxation times the classical Maxwell body. The corresponding creep and relaxation memory functions are expressed in Laplace domain as suitable ratios of modified Bessel functions of contiguous order $\nu>-1$. In time domain these functions are shown to be expressed by Dirichlet series (that is infinite Prony series) so that the corresponding creep compliance and relaxation modulus turn out to be monotonic functions. These functions are characterized by infinite discrete spectra of characteristic times related to the zeros of Bessel functions of the first kind.

The plan of paper is as following. In Section 2 we revisit the essential notions of the linear theory of uni-axial viscoelasticity. The survey will be carried out by adopting the representations of the basic stress - strain relations in the Laplace transform domain, as it is common in linear viscoelasticity. In particular, we will outline the role of the socalled memory functions to characterize the rate of creep and relaxation by using the mathematical notions of completely monotonic functions.

After this introductory discussion, in Section 3 we analyse a general class of fluid-like models (depending on a parameter $\nu>-1$ ) that provides an interesting generalization of the classical Maxwell model. For this purpose we provide the expressions of the Laplace transforms of the memory functions as ratios between of two modified Bessel functions of contiguous orders. The memory functions are then shown to be complete monotonic functions of time, expressed in terms of Dirichlet series. Finally, by a suitable time integration, we derive the corresponding creep compliance and the relaxation modulus (the so-called material functions), so providing a full characterization of the whole class of our viscoelastic models. Due to the relation of these viscoelastic models with the Bessel functions, we agree to refer them to as Bessel models.

Date: August 28, 2018.

Key words and phrases. Viscoelasticity, Creep and relaxation, Bessel functions, Dirichlet series, completely monotone functions. 
In Section 4, for the Bessel models we compute the asymptotic representations of the memory functions and of the corresponding material functions for short and long times. We show some instructive plots of the numerical results in order to outline the time range of validity of the asymptotic representations. We observe that the creep and relaxation properties of our models are consistent with those of a standard Maxwell model for large times. On the other hand, these models are compatible with a fractional Maxwell model of order $1 / 2$, for short times.

Finally, in Section 5 we complete the paper with concluding remarks and hints for future research.

\section{Generalities on Linear viscoelasticity}

According to the linear theory, a viscoelastic body can be considered as a linear system with the stress (or strain) as the excitation function (input) and the strain (or stress) as the response function (output). In this respect, the response functions to an excitation expressed by the Heaviside step function $\Theta(t)$ are known to play a fundamental role both from a mathematical and physical point of view. We denote by $\mathcal{J}(t)$ the strain response to the unit step of stress, according to the creep test and by $\mathcal{G}(t)$ the stress response to a unit step of strain, according to the relaxation test.

The functions $\mathcal{J}(t)$ and $\mathcal{G}(t)$ are usually referred to as the creep compliance and relaxation modulus respectively, or, simply, the material functions of the viscoelastic body. In view of the causality requirement, both functions are vanishing for $t<0$.

The limiting values of the material functions for $t \rightarrow 0^{+}$and $t \rightarrow+\infty$ are related to the instantaneous (or glass) and equilibrium behaviours of the viscoelastic body, respectively. As a consequence, it is usual to denote $\mathcal{J}_{g}:=\mathcal{J}\left(0^{+}\right) \geqslant 0$ the glass compliance, $\mathcal{J}_{e}:=$ $\mathcal{J}(+\infty) \geqslant 0$ the equilibrium compliance, and $\mathcal{G}_{g}:=\mathcal{G}\left(0^{+}\right) \geqslant 0$ the glass modulus $\mathcal{G}_{e}:=$ $\mathcal{G}(+\infty) \geqslant 0$ the equilibrium modulus. As a matter of fact, both the material functions are non-negative with $\mathcal{J}(t)$ non decreasing and $\mathcal{G}(t)$ non increasing.

Denoting by $\sigma(t)$ and $\epsilon(t)$ the uniaxial stress and strain, respectively, and under the hypotheses of sufficiently well behaved causal histories, in most cases the constitutive equations can be written in the following forms

$$
\left\{\begin{aligned}
\epsilon(t) & =\mathcal{J}_{g} \sigma(t)+\int_{0}^{t} \dot{\mathcal{J}}\left(t-t^{\prime}\right) \sigma\left(t^{\prime}\right) d t^{\prime}, \\
\sigma(t) & =\mathcal{G}_{g} \epsilon(t)+\int_{0}^{t} \dot{\mathcal{G}}\left(t-t^{\prime}\right) \epsilon\left(t^{\prime}\right) d t^{\prime}
\end{aligned}\right.
$$

Being of convolution type, equations (2.1) can be conveniently treated by the technique of Laplace transforms so they read in the Laplace domain

$$
\widetilde{\epsilon}(s)=s \widetilde{\mathcal{J}}(s) \tilde{\sigma}(s), \quad \widetilde{\sigma}(s)=s \widetilde{\mathcal{G}}(s) \widetilde{\epsilon}(s),
$$

from which we derive the reciprocity relation

$$
s \tilde{\mathcal{J}}(s)=\frac{1}{s \widetilde{\mathcal{G}}(s)} .
$$

Due to limiting theorems for the Laplace transform, we deduce that

$$
\mathcal{J}_{g}=1 / \mathcal{G}_{g}, \quad \mathcal{J}_{e}=1 / \mathcal{G}_{e},
$$

with the convention that 0 and $+\infty$ are reciprocal to each other. 
The causal functions $\dot{\mathcal{J}}(t)$ and $\dot{\mathcal{G}}(t)$ are referred as the rate of creep (compliance) and the rate of relaxation (modulus), respectively; they play the role of memory functions in the constitutive equations (2.1).

Assuming $\mathcal{J}_{g}>0$ and $\mathcal{G}_{g}>0$, that is restricting our attention to viscoelastic models exhibiting instantaneous elasticity it is convenient to consider the memory functions scaled with their corresponding initial values:

$$
\Psi(t)=\frac{1}{\mathcal{J}\left(0^{+}\right)} \frac{d \mathcal{J}}{d t}, \quad \Phi(t)=-\frac{1}{\mathcal{G}\left(0^{+}\right)} \frac{d \mathcal{G}}{d t} .
$$

These functions are here required to be completely monotonic (CM) functions, that is they are non-negative, non-increasing functions for $t>0$ with infinitely many derivatives alternating in sign. As outlined by several authors, see e.g. [6], [9], the condition of completely monotonicity is sufficient for the physical acceptability and realizability of the models since it ensures, for instance, that in isolated systems the energy decays monotonically as expected from physical considerations. For mathematical details on CM functions we refer the interested reader to the excellent treatise [13].

Then

$$
\begin{aligned}
& \mathcal{J}(t)=\mathcal{J}\left(0^{+}\right)\left[1+\int_{0}^{t} \Psi\left(t^{\prime}\right) d t^{\prime}\right], \\
& \mathcal{G}(t)=\mathcal{G}\left(0^{+}\right)\left[1-\int_{0}^{t} \Phi\left(t^{\prime}\right) d t^{\prime}\right] .
\end{aligned}
$$

Because of the reciprocity relations (2.3) and the above equations, the Laplace transforms of the memory functions are interrelated as follows

$$
1+\widetilde{\Psi}(s)=\frac{1}{1-\widetilde{\Phi}(s)} .
$$

According to their behaviour for large times the viscoelastic models can be considered solid-like (finite creep and relaxation) and fluid-like (infinite creep and complete relaxation), see e.g. [12]. As a consequence for fluid-like models we have

$$
\mathcal{J}(+\infty)=+\infty, \quad \mathcal{G}(+\infty)=0 .
$$

We note the the simplest model of this type is the classical Maxwell body characterized by a single time constant $\tau_{*}$

$$
\Psi(t)=\frac{1}{\tau_{*}}, \quad \Phi(t)=\frac{1}{\tau_{*}} \exp \left(-t / \tau_{*}\right) .
$$

More generally, the fluid-like models exhibit continuous (or discrete) time spectra that we write as

$$
\begin{aligned}
& \Psi(t)=a+b \int_{0}^{\infty} H_{\Psi}(\tau) \exp (-t / \tau) d \tau, \\
& \Phi(t)=c \int_{0}^{\infty} H_{\Phi}(\tau) \exp (-t / \tau) d \tau,
\end{aligned}
$$

with $a, b, c \geqslant 0$ and $H_{\Psi}(\tau) \geqslant 0, H_{\Phi}(\tau) \geqslant 0$. 
It is worth to remark that we can recover the memory functions for the Maxwell model by setting in Eqs. (2.10):

$$
\begin{aligned}
& a=\frac{1}{\tau_{*}}, \quad b=0, \quad H_{\Psi}(\tau) \equiv 0, \\
& c=\frac{1}{\tau_{*}}, \quad H_{\Phi}(\tau)=\delta\left(\tau-\tau_{*}\right),
\end{aligned}
$$

where $\delta$ denotes the Dirac generalized function.

Physically, the mathematical representations (2.10) imply that any processes in linear viscoelasticity can be generally considered as a continuous (or discrete) superposition of elementary (that is exponential) relaxation processes.

\section{The Bessel Models}

In this section we discuss a generalization of the fluid-like model proposed by Giusti and Mainardi in [3] based on modified Bessel functions of order 0,1,2 in the Laplace domain. Indeed, turning to modified Bessel functions of contiguous order $\nu>-1$, we propose a suitable generalization of the memory functions related to the rate of creep and relaxation in the Laplace domain. Consequently, by inversion, we compute both memory functions and the corresponding material functions in the time domain for this class of viscoelastic models that we have agreed to call Bessel models.

3.1. Creep and Relaxation rates. Consider a linear viscoelastic model characterized by a rate of creep function $\Psi_{\nu}(t)$ defined, in the Laplace domain, as

$$
\widetilde{\Psi}_{\nu}(s)=\frac{2(\nu+1)}{\sqrt{s}} \frac{I_{\nu+1}(\sqrt{s})}{I_{\nu+2}(\sqrt{s})}, \quad \nu>-1 .
$$

Now, the Laplace transform of the corresponding rate of relaxation function $\widetilde{\Phi}_{\nu}(t)$ is related to $\widetilde{\Psi}_{\nu}(s)$ as follows

$$
1+\widetilde{\Psi}_{\nu}(s)=\frac{1}{1-\widetilde{\Phi}_{\nu}(s)},
$$

as it is required by the general relation (2.7) between the two memory functions.

Taking into account the recurrence relations for the modified Bessel functions of the first kind $I_{\nu}$, i.e.

$$
I_{\nu-1}(z)-\frac{2 \nu}{z} I_{\nu}(z)=I_{\nu+1}(z),
$$

we immediately get the expression for the Laplace transform of the relaxation memory function $^{1} \Phi_{\nu}(t)$, i.e.

$$
\widetilde{\Phi}_{\nu}(s)=\frac{2(\nu+1)}{\sqrt{s}} \frac{I_{\nu+1}(\sqrt{s})}{I_{\nu}(\sqrt{s})}, \quad \nu>-1 .
$$

Let us first focus on the Laplace Transform of the Rate of Creep $\widetilde{\Psi}_{\nu}(s)$ in Eq. (3.1). We can obtain the Rate of Creep in the time domain by inverting the Laplace transform $\widetilde{\Psi}_{\nu}(s)$. To do this we apply the Bromwich theorem,

$$
\Psi_{\nu}(t)=\frac{1}{2 \pi i} \int_{B r} \widetilde{\Psi}_{\nu}(s) e^{s t} d s
$$

\footnotetext{
${ }^{1}$ For sake of completeness, it is possible to give some functional bounds for these ratios of Bessel functions, in the Laplace domain (see e.g. [8]).
} 
where with $\mathrm{Br}$ we denote the so called Bromwich contour. Now, it is easy to see from the power series of Modified Bessel functions of the First Kind:

$$
I_{\nu}(z)=\left(\frac{z}{2}\right)^{\nu} \sum_{k=0}^{\infty} \frac{1}{k ! \Gamma(\nu+k+1)}\left(\frac{z}{2}\right)^{2 k},
$$

that the function $\widetilde{\Psi}_{\nu}(s)$ is single valued and has a simple pole in $s=0$. Indeed

$$
\begin{aligned}
\widetilde{\Psi}_{\nu}(s) & =\frac{2(\nu+1)}{\sqrt{s}} \frac{I_{\nu+1}(\sqrt{s})}{I_{\nu+2}(\sqrt{s})} \simeq \\
& \simeq \frac{4(\nu+1)(\nu+2)}{s}, \quad \text { as } s \rightarrow 0,
\end{aligned}
$$

where we have made use also of the known property of the Gamma function $\Gamma(z+1)=$ $z \Gamma(z)$. Furthermore, it is straightforward to see that $\widetilde{\Psi}_{\nu}(s)$ has infinite simple poles in $s=-j_{\nu+2, n}^{2}$, where $j_{\nu+2, n}$ represents the $n$th positive real root of the Bessel functions of the corresponding order $J_{\nu+2}(x)=0(n=1,2,3, \ldots)$. Indeed,

$$
I_{\nu+2}(\sqrt{s})=0 \quad \Longleftrightarrow \quad J_{\nu+2}(\lambda)=0,
$$

having renamed $\sqrt{s}=-i \lambda$ with $\lambda>0$ and recalled the known relation $I_{\nu}(i z)=i^{\nu} J_{\nu}(z)$. Therefore, the non-vanishing simple poles for the Laplace transform of the rate of creep are given by

$$
s_{n}=-j_{\nu+2, n}^{2}, \quad n=1,2, \ldots
$$

Now, we can safely compute the Bromwich integral (3.5) by means of the Residues theorem, i.e.

$$
\begin{aligned}
\Psi_{\nu}(t) & =\sum_{s_{n}} \mathcal{R} e s\left\{\widetilde{\Psi}_{\nu}(s) e^{s t} ; s=s_{n}\right\}= \\
& =\sum_{n=0}^{\infty} \mathcal{R} e s\left\{\frac{2(\nu+1)}{\sqrt{s}} \frac{I_{\nu+1}(\sqrt{s})}{I_{\nu+2}(\sqrt{s})} e^{s t} ; s=s_{n}\right\}
\end{aligned}
$$

where $s_{n}=-j_{\nu+2, n}^{2} \neq 0$, for $n \geqslant 1$, and $s_{0}=0$. Thus, we have to distinguish two cases: If $s_{n} \neq 0$, then

$$
\mathcal{R} e s\left\{\widetilde{\Psi}_{\nu}(s) e^{s t} ; s=s_{n}\right\}=4(\nu+1) \exp \left(s_{n} t\right) .
$$

This result is obtained after a few passages hereafter outlined.

$$
\begin{aligned}
& \mathcal{R} e s\left\{\frac{2(\nu+1)}{\sqrt{s}} \frac{I_{\nu+1}(\sqrt{s})}{I_{\nu+2}(\sqrt{s})} e^{s t}\right\}_{s=s_{n}}= \\
& =\lim _{s \rightarrow s_{n}}\left(s-s_{n}\right) \frac{2(\nu+1)}{\sqrt{s}} \frac{I_{\nu+1}(\sqrt{s})}{I_{\nu+2}(\sqrt{s})} e^{s t} \\
& =\lim _{s \rightarrow s_{n}} \frac{2(\nu+1)}{\sqrt{s}} \frac{I_{\nu+1}(\sqrt{s})}{I_{\nu+2}^{\prime}(\sqrt{s}) /(2 \sqrt{s})} e^{s t}= \\
& =4(\nu+1) \exp \left(s_{n} t\right) .
\end{aligned}
$$

Above we have used the property that if $s_{n}$ is a zero of the modified Bessel function $I_{\nu+2}$, then we have,

$$
I_{\nu+2}^{\prime}\left(s_{n}\right)=I_{\nu+1}\left(s_{n}\right),
$$


as we deduce from the general identity for modified Bessel functions, see e.g. [1],

$$
I_{\nu}^{\prime}(z)=-\frac{\nu}{z} I_{\nu}(z)+I_{\nu-1}(z)=\frac{\nu}{z} I_{\nu}(z)+I_{\nu+1}(z) .
$$

Otherwise, if $s=s_{0}=0$, in view of Eq.(3.7)

$$
\begin{aligned}
\mathcal{R} e s\left\{\widetilde{\Psi}_{\nu}(s) e^{s t} ; s=0\right\} & =\lim _{s \rightarrow 0} s \widetilde{\Psi}_{\nu}(s) e^{s t}= \\
& =4(\nu+1)(\nu+2) .
\end{aligned}
$$

Hence, the memory function is given by

$$
\begin{aligned}
\Psi_{\nu}(t)= & 4(\nu+1)(\nu+2)+ \\
& +4(\nu+1) \sum_{n=1}^{\infty} \exp \left(-j_{\nu+2, n}^{2} t\right), \quad \nu>-1,
\end{aligned}
$$

where $j_{\nu+2, n}$ is the $n$th positive real zero of $J_{\nu+2}$ and $t>0$ is the time.

As a consequence, it can be easily noticed that the time representation of the rate of creep $\Psi_{\nu}(t)$ in Eq. (3.15) is a Dirichlet series. In particular, the series in Eq. (3.15) is absolutely convergent for $t>0$. However, we omit the details of this proof that can be easily deduced following the procedures displayed in [3] and [4].

Proceeding in strict analogy with the above discussion, one can easily find an explicit form for the relaxation memory function $\Phi_{\nu}(t)$. Indeed, we find that

$$
\Phi_{\nu}(t)=4(\nu+1) \sum_{n=1}^{\infty} \exp \left(-j_{\nu, n}^{2} t\right),
$$

which is a Dirichlet series absolutely convergent for $t>0$, where $j_{\nu, n}$ is the $n$th positive real zero of $J_{\nu}$ analogously with the discussion above for $\Psi_{\nu}(t)$.

In the particular case $\nu=0$ the memory functions $\Psi_{\nu}$ and $\Phi_{\nu}$ reduce to those dealt in the previous paper [3].

Comparing Eqs. (3.15), (3.16) with Eqs. (2.10) we note that the time spectra of the two memory functions are discrete with characteristic (non-dimensional) times $1 / j_{\nu+2, n}$, $1 / j_{\nu, n}$.

3.2. Material functions. Following the discussion in Section 2, we have that

$$
\begin{aligned}
& s \widetilde{\mathcal{J}}_{\nu}(s)=1+\widetilde{\Psi}_{\nu}(s) \div \mathcal{J}_{\nu}^{\prime}(t)=\Psi_{\nu}(t), \\
& s \widetilde{G}_{\nu}(s)=1-\widetilde{\Phi}_{\nu}(s) \div \mathcal{G}_{\nu}^{\prime}(t)=-\Phi_{\nu}(t),
\end{aligned}
$$

by setting for convenience $\mathcal{J}_{\nu}\left(0^{+}\right)=\mathcal{G}_{\nu}(0+)=1$.

Hence we get

$$
\begin{aligned}
& \mathcal{J}_{\nu}(t)=1+\int_{0}^{t} \Psi_{\nu}(u) d u, \\
& \mathcal{G}_{\nu}(t)=1-\int_{0}^{t} \Phi_{\nu}(u) d u .
\end{aligned}
$$

Integrating term by term the Dirichlet series defining $\Psi_{\nu}(t)$ and $\Phi_{\nu}(t)$ we get

$$
\begin{aligned}
\mathcal{J}_{\nu}(t) & =1+4(\nu+1)(\nu+2) t+4(\nu+1) \times \\
& \times \sum_{n=1}^{\infty}\left[\frac{1}{j_{\nu+2, n}^{2}}-\frac{1}{j_{\nu+2, n}^{2}} \exp \left(-j_{\nu+2, n}^{2} t\right)\right],
\end{aligned}
$$




$$
\mathcal{G}_{\nu}(t)=1-4(\nu+1) \sum_{n=1}^{\infty}\left[\frac{1}{j_{\nu, n}^{2}}-\frac{1}{j_{\nu, n}^{2}} \exp \left(-j_{\nu, n}^{2} t\right)\right]
$$

Now, if we recall the following result concerning the zeros of the Bessel functions of the first kind (see e.g. [14], [4])

$$
\sum_{n=1}^{\infty} \frac{1}{j_{\nu, n}^{2}}=\frac{1}{4(\nu+1)},
$$

then we get

$$
\begin{aligned}
& \mathcal{J}_{\nu}(t)= 2\left(\frac{\nu+2}{\nu+3}\right)+4(\nu+1)(\nu+2) t \\
&-4(\nu+1) \sum_{n=1}^{\infty} \frac{1}{j_{\nu+2, n}^{2}} \exp \left(-j_{\nu+2, n}^{2} t\right) \\
& \mathcal{G}_{\nu}(t)=4(\nu+1) \sum_{n=1}^{\infty} \frac{1}{j_{\nu, n}^{2}} \exp \left(-j_{\nu, n}^{2} t\right) .
\end{aligned}
$$

\section{Numerical RESUlts AND ASYMPtotic REPRESENTATIONS}

For our Bessel models we now exhibit versus non-dimensional time the plots of the creep and relaxation memory functions $\Psi_{\nu}(t), \Phi_{\nu}(t)$ according to Eqs. (3.15), (3.16), and of the corresponding material functions $\mathcal{J}_{\nu}(t)$ and $\mathcal{G}_{\nu}(t)$ according to Eqs. (3.22), (3.23). The corresponding Dirichlet series have been computed by taking 100 terms to ensure a suitable numerical convergence. We have used an efficient MATLAB routine given in [11] to compute the requested zeros of the Bessel functions. In order to have an idea of the effect of the parameter $\nu>-1$ we have compared the plots selecting the values $\nu=-0.5,0,0.5,1$, and using suitable logarithmic scales.

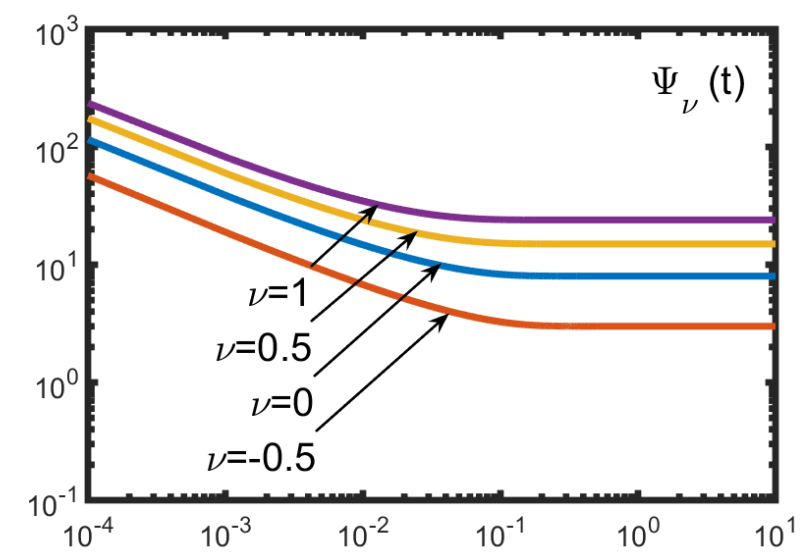

Fig.1: The creep-memory function $\Psi_{\nu}(t)$ for $\nu=-0.5,0,0.5,1$. 


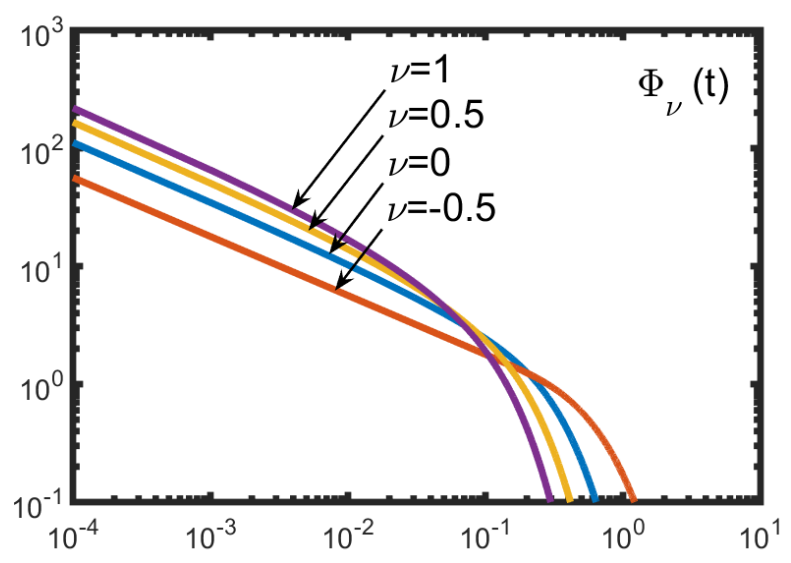

Fig.2: The relaxation-memory function $\Phi_{\nu}(t)$ for $\nu=-0.5,0,0.5,1$.

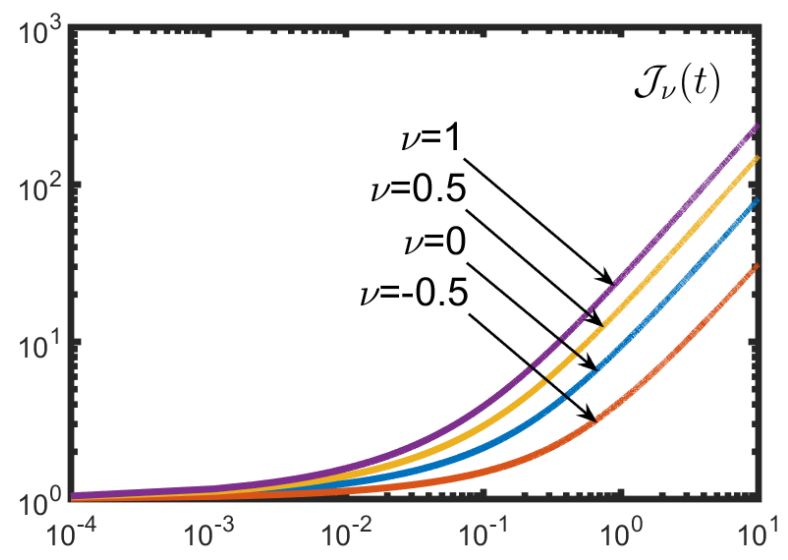

Fig.3: The creep compliance $\mathcal{J}_{\nu}(t)$ for $\nu=-0.5,0,0.5,1$.

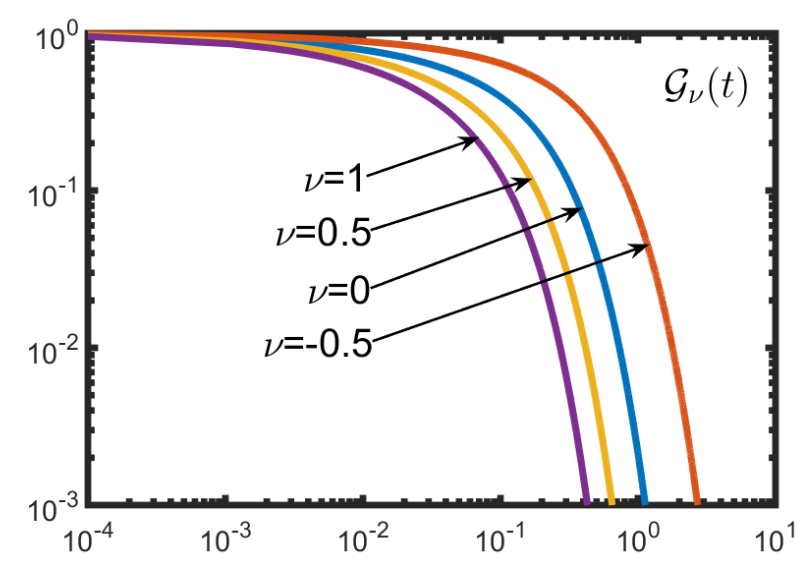

Fig.4: The relaxation modulus $\mathcal{G}_{\nu}(t)$ for $\nu=-0.5,0,0.5,1$.

We are also interested to see how the asymptotic representations of the memory and material functions for short and long time are matching with the corresponding numerical solutions. For sake of brevity we limit ourselves to consider the case $\nu=1$ that would 
provide a sufficient overview of the matching. We remark that the case $\nu=0$ was already considered in the recent paper by Giusti and Mainardi [3].

The asymptotic representations of the memory functions for short times are formally derived according to a Tauberian theorem by inverting their Laplace transforms (3.1), (3.4) approximated as $s \rightarrow \infty$. For this purpose we use the known asymptotic representation of the generic modified Bessel function

$$
I_{\nu}(z) \sim \frac{e^{z}}{\sqrt{2 \pi} z^{1 / 2}}, \quad|z| \rightarrow \infty,|\arg z|<\frac{\pi}{2}
$$

For long times it is sufficient to take the first term of the corresponding expressions (3.15), (3.16). Finally the asymptotic representations read:

$$
\Psi_{\nu}(t) \sim \begin{cases}\frac{2(\nu+1)}{\sqrt{\pi}} t^{-1 / 2}, & t \rightarrow 0, \\ 4(\nu+1)(\nu+2), & t \rightarrow \infty,\end{cases}
$$

and

$$
\Phi_{\nu}(t) \sim \begin{cases}\frac{2(\nu+1)}{\sqrt{\pi}} t^{-1 / 2}, & t \rightarrow 0 \\ 4(\nu+1) \exp \left(-j_{\nu, 1}^{2} t\right), & t \rightarrow \infty\end{cases}
$$

In the subsequent Figures 5-6-7-8. we show in continuous lines the plots of the memory and material functions versus time, in comparison with the corresponding asymptotic representations for short and long times, printed with dashed and dotted lines, respectively.

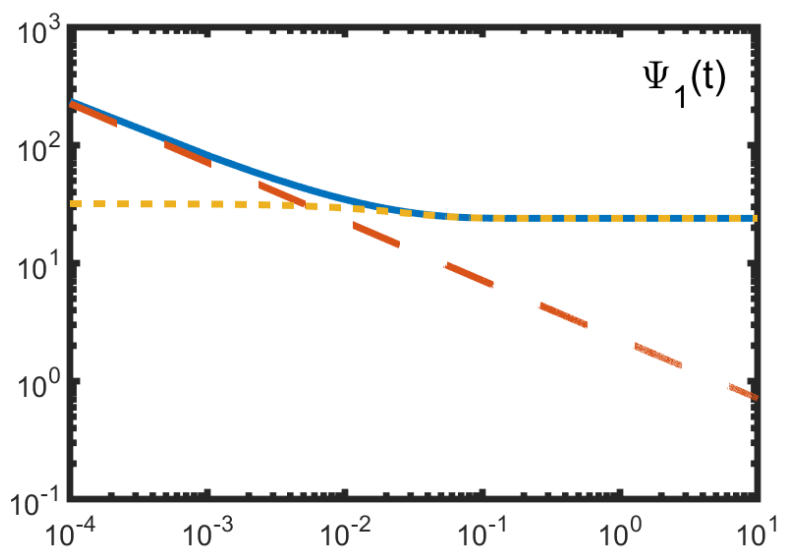

Fig.5: The memory function $\Psi_{1}(t)$ in comparison with its asymptotic approximations. 


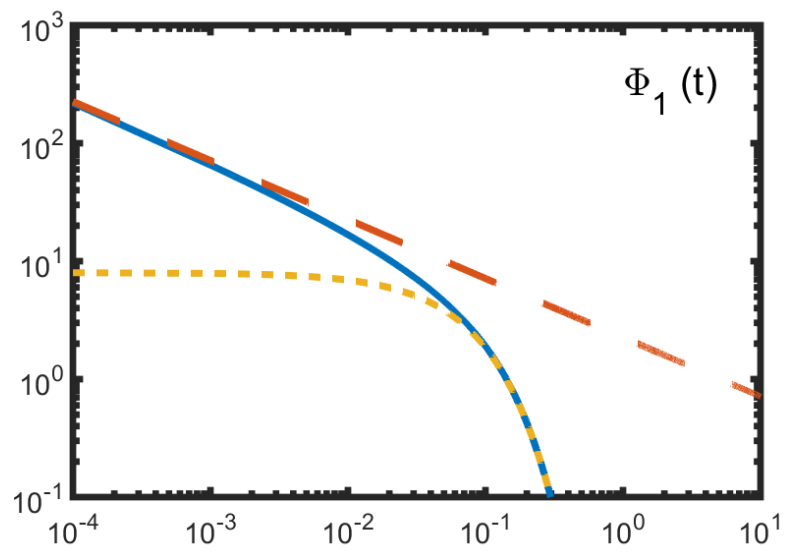

Fig.6: The memory function $\Phi_{1}(t)$ in comparison with its asymptotic approximations.

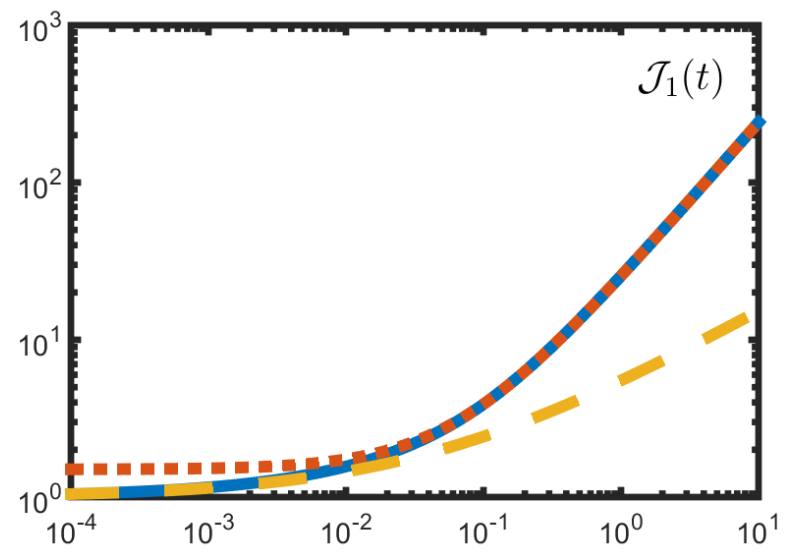

Fig.7: The creep compliance $\mathcal{J}_{1}(t)$ in comparison with its asymptotic approximations.

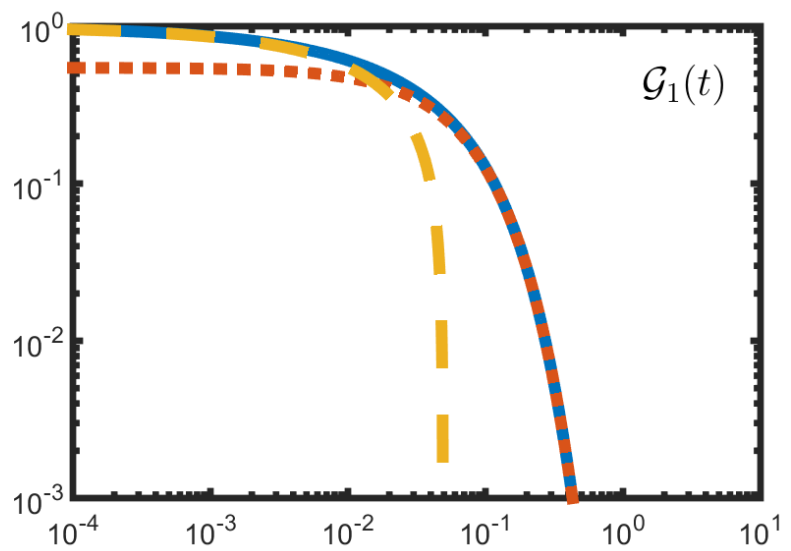

Fig.8: The relaxation modulus $\mathcal{G}_{1}(t)$ in comparison with its asymptotic approximations. 
The asymptotic representations of $\mathcal{J}_{\nu}(t)$ and $\mathcal{G}_{\nu}(t)$ are easily derived from Eqs. (3.18) by integrating $\Psi_{\nu}(t)$ and $\Phi_{\nu}(t)$ given by $(4.2),(4.3)$, respectively. They read:

$$
\mathcal{J}_{\nu}(t) \sim \begin{cases}1+\frac{4(\nu+1)}{\sqrt{\pi}} t^{1 / 2}, & t \rightarrow 0, \\ 2 \frac{(\nu+2)}{(\nu+3)}+4(\nu+1)(\nu+2) t, & t \rightarrow \infty\end{cases}
$$

and

$$
\mathcal{G}_{\nu}(t) \sim \begin{cases}1-\frac{4(\nu+1)}{\sqrt{\pi}} t^{1 / 2}, & t \rightarrow 0, \\ 4(\nu+1) \frac{1}{j_{\nu, 1}^{2}} \exp \left(-j_{\nu, 1}^{2} t\right), & t \rightarrow \infty,\end{cases}
$$

It is interesting to show in Table I the numerical values of the first positive zero $j_{\nu, 1}$ of the Bessel function $J_{\nu}$ and of its square for the 4 cases of $\nu$ considered in the plots. This way we get an hint on how the exponential decay in relaxation behaves as $\nu$ increases, see Eqs. (4.3), (4.5).

\begin{tabular}{|c|c|c|c|c|}
\hline$\nu$ & -0.5 & 0 & 0.5 & 1 \\
\hline$j_{\nu, 1}$ & 1.57 & 2.40 & 3.14 & 3.83 \\
\hline$j_{\nu, 1}^{2}$ & 2.47 & 5.78 & 9.87 & 14.68 \\
\hline
\end{tabular}

Table I

Based on the above asymptotic results and on the theory of fractional viscoelastic models dealt with detail in $[9 ; 10]$, we recognize that the creep and relaxation properties of the viscoelastic models under consideration are compatible with those of a peculiar model. In particular, they are consistent with a fluid-like body, that, for short times, is akin with a fractional Maxwell model of order $1 / 2$ and, for large times, is similar to a standard Maxwell model.

\section{Conclusions AND Final REMARKS}

In this paper we have illustrated a parametric class of linear viscoelastic models of fluid-like type depending on a parameter $\nu>-1$. In particular, for $\nu=0$ we recover the model recently introduced by Giusti and Mainardi [3] for describing the effect of fluid viscosity for fluid filled elastic tubes. In the Laplace domain the memory and material functions characterizing this class are represented by ratios of modified Bessel functions of contiguous order $\nu$, so that we agree to refer them to as Bessel models. In analogy with the case $\nu=0$, the models of this general class exhibit (with a different strength and time range depending on $\nu$ ) the creep and relaxation properties typical of a fractional Maxwell body of order $1 / 2$ for short times, and of a standard Maxwell body, for long times. We have noted from the plots in Figs 1-4 that the time range of validity of short time approximation is shorter as higher is the value of the parameter $\nu$. This effect appears clear from inclined (parallel) straight lines in log-log scales.

However, this asymptotic behavior of power law type with exponent $\pm 1 / 2$ exhibited by the memory and material functions, even though for a restricted range of time after the initial instant $t=0$, is peculiar of the whole class of Bessel models.

The above analysis is worth to be pursued in future with respect to the evolution of the transient waves propagating through viscoelastic media modelled by this class. Indeed, as formerly pointed out in 1975 by Buchen and Mainardi in [2] (see also [9]), the singular behavior of the memory and material functions at $t=0^{+}$induces a wave-front smoothing 
of any initial discontinuity, akin to a diffusion effect. For more details on this smoothing effect found in transient waves propagating in media with singular memory, we refer to Hanyga [5].

It is also interesting to stress that such models could be of a certain interests for the study of physical properties of polymers. Indeed, this class of models could turn out to be useful to describe polymers featuring dynamical behavior akin to the one described above. In such cases, the value for $\nu$ could then be appropriately tuned in order to fit the experimental data.

\section{ACKNOWLEDGEMENTS}

The work of A. G. and F. M. has been carried out in the framework of the activities of the National Group of Mathematical Physics (GNFM, INdAM).

\section{REFERENCES}

[1] M. Abramowitz and I.A. Stegun, Handbook of Mathematical Functions, Dover, New York (1965).

[2] P.W. Buchen, F. Mainardi, Asymptotic expansions for transient viscoelastic waves, Journal de Mécanique 14, 597-608 (1975).

[3] A. Giusti, F. Mainardi, A dynamic viscoelastic analogy for fluid-filled elastic tubes, Meccanica, published on line, 04 February 2016. DOI: 10.1007/s11012-016-0376-4 E-print, arXiv:1505.06695 (2015).

[4] A. Giusti, F. Mainardi, On infinite series concerning zeros of Bessel functions of the first kind, E-print, arXiv:1601.00563 (2016).

[5] A. Hanyga, Wave propagation in media with singular memory, Mathematical and Computer Modelling 34, 1399-1421 (2001).

[6] A. Hanyga, Physically acceptable viscoelastic models, in Hutter, K. and Wang, Y. (Editors), Trends in Applications of Mathematics to Mechanics, Shaker Verlag GmbH, Aachen, pp. 125-136 (2005).

[7] G.H. Hardy, M. Riesz, The General Theory of Dirichlet Series, Cambridge University Press, Cambridge (1915).

[8] A. Laforgia, P. Natalini, Some Inequalities for Modified Bessel Functions, Journal of Inequalities and Applications, Volume 2010, Article ID 253035, 10 pages.

[9] F. Mainardi, Fractional Calculus and Waves in Linear Viscoelasticity, Imperial College Press, London (2010).

[10] F. Mainardi, G. Spada, Creep, relaxation and viscosity properties for basic fractional models in rheology, Eur. Phys. J. Special Topics 193, 133-160 (2011). E-print, arXiv:1110.3400 (2011).

[11] MATLAB Central, File Exchange, Bessel Function Zeros by Greg von Winckel, File ID: \#6794 (Updated 27 Jan 2005)

[12] A.C. Pipkin, Lectures on Viscoelastic Theory, Springer-Verlag, New York (1986).

[13] R.L. Schilling, R. Song, Z. Vondracek, Bernstein Functions. Theory and Applications, 2-nd Edition, De Gruyter, Berlin (2012).

[14] I. N. Sneddon, On some infinite series involving the zeros of Bessel functions of the first kind, Proc. Glasgow Math. Assoc. 4, 144-156 (1960). 
${ }^{1}$ Department of Physics \& Astronomy, University of Bologna and infN. Via Irnerio 46, BOLOGNA, ITALY.

E-mail address: ivano.colombaro@bo.infn.it

2 Department of Physics \& Astronomy, University of Bologna and infN. Via Irnerio 46, BOLOGNA, ITALY.

E-mail address: andrea.giusti@bo.infn.it

${ }^{3}$ Department of Physics \& Astronomy, University of Bologna and infN. Via Irnerio 46, BOLOGNA, ITALY.

E-mail address: francesco.mainardi@bo.infn.it 